\title{
Correlated responses in death-feigning behavior, activity, and brain biogenic amine expression in red flour beetle Tribolium castaneum strains selected for walking distance
}

\author{
Kentarou Matsumura $^{1} \cdot$ Ken Sasaki $^{2} \cdot$ Takahisa Miyatake $^{1}$
}

Received: 12 July 2015/ Accepted: 14 November 2015/Published online: 8 December 2015

(C) Japan Ethological Society and Springer Japan 2015

\begin{abstract}
Dispersal ability may influence antipredator and mating strategies. A previous study showed a tradeoff between predation avoidance and mating success in strains of the red flour beetle Tribolium castaneum selected for walking distance. Specifically, beetles derived from strains selected for longer walking distance suffered higher predation pressure and had higher male mating success than their counterparts derived from strains selected for shorter walking distance. In the study reported here, we compared the locomotor activity, biogenic amine expression in the brain, and death-feigning behavior of the red flour beetle strains selected for walking distance. The results indicated that individuals genetically predisposed to longer walking distance had higher locomotor activity and lower intensity of deathfeigning behavior than those genetically predisposed to shorter walking distance. However, no significant differences were found in the expression of biogenic amines in the brain among strains selected for walking distance, although the level of dopamine in the brain differed from that of the strains divergently selected for duration of death-feigning behavior. The relationships between walking speed, activity, death-feigning behavior, and brain biogenic amines in $T$. castaneum are discussed.
\end{abstract}

Takahisa Miyatake

miyatake@cc.okayama-u.ac.jp

1 Laboratory of Evolutionary Ecology, Graduate School of Environmental and Life Science, Okayama University,

Tsushima-Naka, Okayama City, Okayama, Japan

2 Department of Bioresource Science, Tamagawa University, Machida, Tokyo, Japan
Keywords Artificial selection - Biogenic amine $\cdot$ Deathfeigning behavior - Dispersal ability · Genetic correlation . Locomotor activity

\section{Introduction}

The dispersal of animals is an essential feature of survival (Clobert et al. 2009; Bonte et al. 2012). In many animals, dispersal ability is closely related to a suite of other traits, referred to collectively as the dispersal syndrome (Frazzetta 1975; Clobert et al. 2009). For example, in some insect species with wing size dimorphism, individuals with longer wings and greater flight ability show lower reproductive success because more resources are invested in wing size than in ovary size. In contrast, short-winged individuals invest their resources in increasing ovary size, resulting in an increase in reproductive success (Roff and Fairbairn 2007). Both dispersal and non-dispersal types are frequently found in natural populations due to a resource allocation trade-off, and this can help to explain the maintenance of variation in dispersal ability within a population (reviewed in Roff and Fairbairn 2007; Bonte et al. 2012).

Predation is a major selection pressure in shaping prey behavior (Lima and Dill 1990), and because natural selection favors individuals that successfully avoid predators, evolution should favor the adaptive antipredator behavior of prey. Dispersal often increases the risk of predation due to the increased frequency of encounter with predators (e.g., Aukema and Raffa 2004; Korb and Linsenmair 2002; Srygley 2004; Matsumura and Miyatake 2015), which suggests that prey should evolve antipredator strategies that are dependent on its own dispersal ability. When a prey perceives danger, it often uses two alternative tactics to survive-flight or fight, or running or death-feigning (e.g., 
Martin and Lopez 2000; Lehtiniemi 2005; Wasson and Lyon 2005; Ohno and Miyatake 2007). Individuals with higher dispersal ability may adopt a running escape tactic to survive when they encounter a predator because they have a higher ability to move and thus avoid the predator. In contrast, individuals with lower dispersal ability may adopt a death-feigning tactic to survive when they encounter a predator due to their lower ability to move.

Death-feigning behavior (sometimes referred to as thanatosis) is considered to be a defense mechanism against predators that has evolved in many animals, and its adaptive significance has been reported in many studies (e.g. Edmunds 1974; Miyatake et al. 2004; Ruxton et al. 2004). Miyatake et al. (2004) carried out artificial selection for duration of death-feigning in the red flour beetle Tribolium castaneum and reported that individuals genetically predisposed for longer duration of death-feigning (LD strain) were more successful in surviving an encounter with the jumping spider Hasarius adansoni than their counterparts with a genetic predisposition for shorter duration of death-feigning (SD strain). These authors also observed that the walking distance of beetles derived from LD strains was shorter than that of SD strains and that the expression of brain dopamine in LD strains was significantly lower than that in SD strains (Miyatake et al. 2008a).

Many insect behaviors, including aggressiveness and activity, are controlled by biologically active substances, including biogenic amines (e.g., Evans 1980; Bicker and Menzel 1989; Stevenson et al. 2000). In the sand cricket Gryllus firmus, dispersal ability is controlled in part by juvenile hormone, suggesting that in this species dispersal and physiological mechanism may be closely related to each other (reviewed in Roff and Fairbairn 2007). However, few studies to date have investigated the relationship between dispersal ability and biogenic amines (e.g., Miyatake et al. 2008a).

Matsumura and Miyatake (2015) recently performed artificial selection for walking distance, i.e., an indicator of dispersal ability, in the red flour beetle $T$. castaneum and established strains with longer (LW strain) or shorter (SW strain) walking distances. They also compared the frequency of predation by the assassin bug Amphibolus venator, reporting that $\mathrm{LW}$ strain beetles suffered higher predation risk than did SW strain beetles (Matsumura and Miyatake 2015). If dispersal ability is genetically correlated with antipredator strategies, two-way artificial selection for dispersal ability should show correlated responses in the expression of biogenic amines and death-feigning behavior. In the study reported here, we compared deathfeigning and expression of biogenic amines of strains selected for longer or shorter walking distance (LW vs. $\mathrm{SW})$. We also examined the locomotor activity of these two selected strains to test whether walking distance correlates with locomotor activity.

\section{Materials and methods}

\section{Insect}

The red flour beetles (Tribolium castaneum) used in this study have been maintained in laboratories for more than 30 years. The beetles were reared on a mixture of wholemeal (Yoshikura Shokai, Tokyo, Japan) enriched with brewer's yeast (Asahi Beer, Tokyo, Japan) in an incubator (Sanyo, Tokyo, Japan) maintained at $25{ }^{\circ} \mathrm{C}$ under a $16: 8$-h (light:dark) photoperiod (lights on at 0700 hours; lights off at 2300 hours). They were sexed based on distinct morphological characters of the abdomen in the pupa stage and stored in single-sex groups in a dish (diameter $90 \mathrm{~mm}$, height $15 \mathrm{~mm}$ ) until the experiments.

\section{Artificial selection}

Seventy-five virgin males and 75 virgin females (21-28 days old) were randomly collected from a stock culture, and the walking distance of each beetle was measured by an image tracker (Digimo, Osaka, Japan) for $30 \mathrm{~min}$. The ten males and ten females with the longest walking distance $(13.3 \%)$ were selected to propagate the relatively long-distance walking strain (LW strain). Similarly, the ten males and ten females with the shortest walking distance $(13.3 \%)$ were selected to propagate the relatively short-distance walking strain (SW strain). This procedure was repeated at each generation for 19 generations. Three replicate lines were produced for each strain. More detailed information on the artificial selection process, including direct responses until the 15th generation, is provided by Matsumura and Miyatake (2015).

\section{Walking speed}

For the walking speed experiment, 75 virgin males and 75 virgin females (21-28 days old) of the 17th and 18th generation were randomly collected from each selection strain. One day before the experiment, each beetle was placed in a well of a 48-well tissue culture plate (CELLSTAR $\AA$; Greiner Bio One, Frickenhauser, Germany). The walking distance of each beetle was measured the next day by the method described above, and the moving speed per minute was calculated.

\section{Death-feigning behavior}

In this experiment, 50 virgin males and 50 virgin females (21-28 days old) of the 17th and 18th generations were randomly collected from each selection strain and the duration of death-feigning behavior measured. One day before the observation, each beetle was placed in a well of 
a 48-well tissue culture plate to prevent disturbance by other beetles, which usually reduces the duration of deathfeigning (Miyatake 2001). In the experiment conducted the day thereafter, each of the beetles was gently placed on its back in a white china saucer (diameter $140 \mathrm{~mm}$, height $15 \mathrm{~mm}$ ), and death-feigning behavior was induced by touching the abdomen of the beetle with a wooden stick. A trial consisted of provoking the death-feigning behavior and recording its duration with a stopwatch. The duration of the behavior was defined as the length of time between the stick touching the beetle and detection of its first visible movement. If the beetle did not respond, the touch was repeated. When the beetle feigned death, the duration was recorded. All trials were conducted between 1200 hours and 1700 hours in a chamber (Sanyo) maintained at $25{ }^{\circ} \mathrm{C}$ and $60 \%$ humidity, under a 16-h (light) photoperiod (lights on at 0700 hours, lights off at 2300 hours).

\section{Locomotor activity}

Each beetle (male: $N=20 \times$ sex $\times$ two strains $\times$ three replicate lines; female: $N=23 \times$ sex $\times$ two strains $\times$ three replicate lines) was placed in a separate well of a 48-well culture plate for 1 day, and then put into a petri dish without rearing medium. Locomotor activity was monitored using an infrared actograph. A photomicrosensor (E3R-5E4/R2E4DS30E4; Omron Corp., Kyoto, Japan) was situated at the midpoint of the dish, with the emitter and detector devices placed on either side of the dish. When the beetle passed the midpoint of the dish, the infrared light was interrupted. The beetle was kept in dish for $2 \mathrm{~h}$ for acclimatization, and then the numbers of interruptions of the infrared light were recorded for $22 \mathrm{~h}$ as an index of locomotor activity. All trials were conducted in an incubator (Sanyo) maintained at $25^{\circ} \mathrm{C}$ in the dark.

\section{Biogenic amine}

Expressions of brain biogenic amines in T. castaneum were measured. In these experiments, we used the beetles from only the third replicate line as these individuals showed a well-diverged response to selection (see Fig. 1). Octopamine (OA), $N$-acetyldopamine (NADA), dopamine (DA), and 5-hydroxytryptamine (5HT) content in the brains of beetles of the 18th generation of the selected LW and SW strains, respectively, were measured. OA, NADA, DA, and 5HT content was also measured in beetles of the 19th generation of $\mathrm{LW}, \mathrm{SW}$, and $\mathrm{C}$ strains (i.e., randomly chosen from stock cultures).

Whole bodies of adult $T$. castaneum males were frozen and stored in liquid nitrogen until dissection. The brain tissue without the optic lobes, frontal ganglion, and subesophageal ganglion was removed from the frozen head with
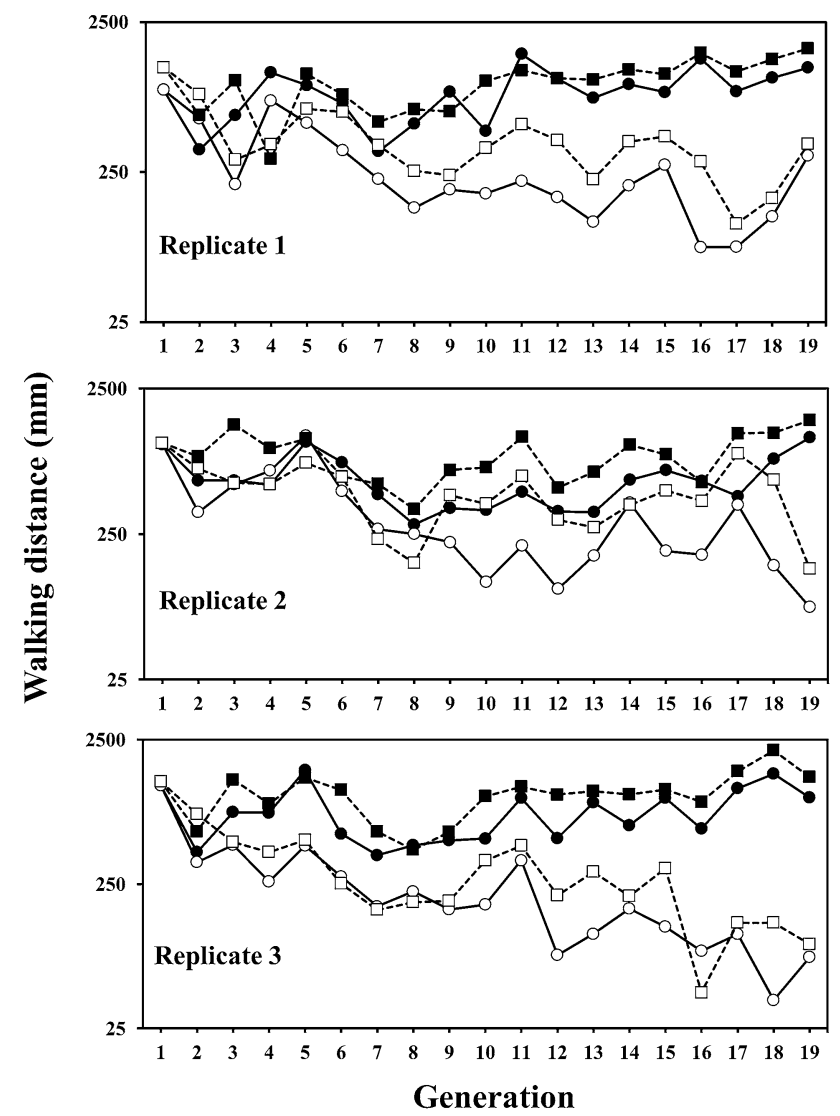

Fig. 1 Direct response to artificial selection for walking distance in 19 generations of the red flour beetle Tribolium castaneum. Filled symbols long-distance walking (LW) strain, open symbols shortdistance walking (SW) strain, circles males squares females. Data between generation 1 and 15 were derived from Matsumura and Miyatake (2015). For more detailed data on these generations, see Matsumura and Miyatake (2015, Electronic Supplementary Material S1)

fine tweezers and a dissecting needle. During dissection, the tissue was immersed in ice-cold $0.1 \mathrm{M}$ phosphate buffer (pH 7.0) on a Peltier cooling unit (Kenis Ltd., Osaka, Japan) at approximately $4{ }^{\circ} \mathrm{C}$. The tissue collected from five heads was homogenized with a glass microhomogenizer in $50 \mu \mathrm{l}$ of ice-cold $0.1 \mathrm{M}$ perchloric acid containing $12.5 \mathrm{ng} / \mathrm{ml}$ 3,4-dihydroxybenzylamine as the internal standard. The sample was centrifuged at $20,600 \mathrm{~g}$ for $30 \mathrm{~min}$ at $4{ }^{\circ} \mathrm{C}$. The supernatant was transferred to a micro-vial for subsequent high-performance liquid chromatography-electrochemical detector (HPLC-ECD) analysis.

An improved version of the method described by Sasaki et al. (2007) and Matsuyama et al. (2015) was used to quantify biogenic amines. The HPLC-ECD system consisted of a solvent delivery pump, refrigerated automatic injector (AS-2057; JASCO Corp., Tokyo, Japan), and a C18 reversed-phase column (UG 120 U column: $250 \times 4.6 \mathrm{~nm}$ i.d., average particle size $5 \mathrm{~mm}$; Shiseido, Tokyo, Japan) maintained at $35{ }^{\circ} \mathrm{C}$ in a column oven. An 
electrochemical detector (ECD-300; Eicom Corp., Kyoto, Japan) with a glassy carbon electrode set at $0.83-0.84 \mathrm{~V}$ was used. Signals from the electrochemical detector were recorded and integrated using data analysis software (PowerChrom; eDAQ Pty Ltd., Denistone East, NSW, Australia). The mobile phase contained $0.18 \mathrm{M}$ monochloroacetic acid and $40 \mathrm{mM}$ of Na2EDTA adjusted to $\mathrm{pH}$ 3.6 with $\mathrm{NaOH}$. Both $2.77 \mathrm{mM}$ of sodium-1-octanesulfonate and $5 \% \mathrm{CH}_{3} \mathrm{CN}$ were added to this solution. The flow rate was kept constant at $0.7 \mathrm{ml} / \mathrm{min}$. External standards were run before and after sample runs to identify and quantify the biogenic amines. A peak of the biogenic amines was identified by comparing both the retention time and hydrodynamic voltamograms with the standards. Measurements were obtained by comparing peak areas of samples and standard chromatograms.

\section{Statistical analysis}

To compare the duration and frequency of death-feigning, locomotor activity, and walking speed of the LW and SW strains, we used the restricted maximum likelihood (REML) approach, with selected strains and sex as fixed effects and replicate lines as a random effect. The duration of death-feigning $( \pm 1 \mathrm{~s})$ was then log-transformed to approximate normality. For comparison of the expression of biogenic amines, one-way analysis of variance (ANOVA) was used. All statistics were analyzed by JMP version 7 (SAS 2007).

\section{Results}

\section{Direct response in walking distance}

The direct response to selection for walking distance over 19 generations in shown in Fig. 1. The walking distance was divided clearly between the two selection regimes in three replicates. Table 1 shows the results of the statistical analysis for walking distance at the 17th and 18th generation by REML. LW strains walked a significantly longer distance than SW strains, and females walked a longer distance than males. We found that the interactions selected strain $\times$ sex and selected strain $\times$ generation significantly affected walking distance in the two strains. Other interactions were not found to be significant.

\section{Death-feigning behavior}

The frequency of death-feigning of the selected strains is shown in Fig. 2. The beetles from LW strains showed a significantly lower frequency of death-feigning than SW strains at generations 17 and 18 (see also Table 1). The
Table 1 Walking distance and intensity (frequency and duration) of death-feigning behavior by selected Tribolium castaneum strains according to restricted maximum likelihood ${ }^{\mathrm{a}}$

\begin{tabular}{|c|c|c|c|}
\hline Source & $d f$ & $F$ & $P$ \\
\hline \multicolumn{4}{|l|}{ Walking distance } \\
\hline Selected strain & 1 & 798.916 & $<0.0001 *$ \\
\hline Sex & 1 & 73.1232 & $<0.0001 *$ \\
\hline Generation & 1 & 3.5812 & 0.0586 \\
\hline Selected strain $\times$ sex & 1 & 10.2667 & $0.0014 *$ \\
\hline Selected strain $\times$ generation & 1 & 19.09 & $<0.0001^{*}$ \\
\hline Sex $\times$ generation & 1 & 0.897 & 0.3437 \\
\hline Selected strain $\times \operatorname{sex} \times$ generation & 1 & 0.7074 & 0.4004 \\
\hline \multicolumn{4}{|l|}{ Frequency of death-feigning behavior } \\
\hline Selected strain & 1 & 180.03 & $<0.0001 *$ \\
\hline Sex & 1 & 0.51 & 0.4750 \\
\hline Generation & 1 & 5.30 & $0.0215 *$ \\
\hline Selected strain $\times$ sex & 1 & 1.07 & 0.3022 \\
\hline Selected strain $\times$ generation & 1 & 1.82 & 0.1774 \\
\hline Sex $\times$ generation & 1 & 6.86 & $0.0089^{*}$ \\
\hline Selected strain $\times \operatorname{sex} \times$ generation & 1 & 5.30 & 0.2150 \\
\hline \multicolumn{4}{|l|}{ Duration of death-feigning behavior } \\
\hline Selected strain & 1 & 578.109 & $<0.0001 *$ \\
\hline $\operatorname{Sex}$ & 1 & 11.0414 & $0.0009 *$ \\
\hline Generation & 1 & 2.2719 & 0.1320 \\
\hline Selected strain $\times$ sex & 1 & 0.2978 & 0.5853 \\
\hline Selected strain $\times$ generation & 1 & 9.0097 & $0.0027 *$ \\
\hline Sex $\times$ generation & 1 & 8.3348 & $0.0040^{*}$ \\
\hline Selected strain $\times \operatorname{sex} \times$ generation & 1 & 2.6056 & 0.1068 \\
\hline
\end{tabular}

* Significant at $P<0.05$

${ }^{\text {a }}$ In this statistical analysis, selected strain and sex were fixed effects and replicate line was the random effect

REML results (Table 1) indicate that sex did not have a significant effect on death-feigning but that the interaction of sex $\times$ generation did have a significant effect. The effects of other interactions were non-significant.

Figure 3 shows the duration of death-feigning of the selected strains (see Table 1 for REML results). The beetles from LW strains showed significantly shorter duration of death-feigning than those from SW strains, and males showed a longer duration of death-feigning than females. The REML results indicate that the effects of the selected strain $\times$ generation and sex $\times$ generation interactions were significant. The effects of all other interactions were nonsignificant effect.

\section{Locomotor activity}

The locomotor activity of the selected strains at the 16th generation is shown in Fig. 4. The beetles from the LW strains showed significantly higher activity than those of 



Fig. 2 Frequency of death-feigning behavior of $T$. castaneum LW strains (filled bar) and SW strains (open bar) at the 17th and 18th generations

the SW strains $(F=40.24, P<0.0001)$, but the effects of other interactions were non-significant (sex: $F=1.28$, $P=0.2586$; strain $\times$ sex: $F=0.07, P=0.7797)$.

\section{Biogenic amines}

The results of the statistical analysis for brain biogenic amine expression at the 18th and 19th generations in the third replicate line are shown in Table 2. ANOVA showed that in terms of expression of the biogenic amines evaluated in the study, there were no significant differences between the selected strains (Table 2).

\section{Walking speed}

Walking speeds of the selected strains are shown in Fig. 5. Beetles from the LW strains had a significantly higher walking speed than those from the SW strains ( $F=798.92, P<0.0001)$. Females showed a significantly higher walking speed than males $(F=73.12, P<0.0001)$. Significant effects of interaction were found for selected


Fig. 3 Duration of death-feigning behavior of T. castaneum LW strains (filled bars) and SW strains (open bars) at 17th and 18th generations. Error bars Standard error (SE)

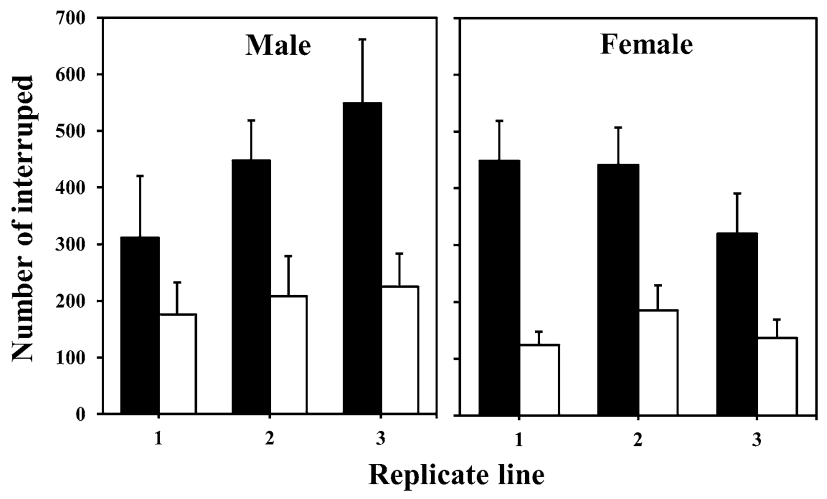

Fig. 4 Locomotor activity of T. castaneum LW strains (filled bars) and SW strains (open bars) at the 16th generation. Error bars SE

strain $\times$ generation $(F=19.09, P<0.0001)$ and selected strain $\times \operatorname{sex}(F=10.27, P=0.0014)$. Significant effects were not found for other interactions (generation: $F=3.58, \quad P=0.0586 ; \quad$ sex $\times$ generation: $\quad F=0.90$, $P=0.3437 ; \quad$ strain $\times$ sex $\times$ generation: $\quad F=0.71$, $P=0.4004)$. 
Table 2 Results of analysis of variance on the expression of brain biogenic amines by the selection strains of T. castaneum at the 18th and 19th generations

\begin{tabular}{|c|c|c|c|c|c|c|}
\hline \multirow[t]{2}{*}{ Generation } & \multirow[t]{2}{*}{ Amines } & \multicolumn{3}{|l|}{ T. castaneum strains $^{\mathrm{a}}$} & \multirow[t]{2}{*}{$F$} & \multirow[t]{2}{*}{$P$} \\
\hline & & LW (pmol/sample) & SW (pmol/sample) & Control (pmol/sample) & & \\
\hline \multirow[t]{4}{*}{ 18th } & OA & $2.407 \pm 0.52$ & $2.392 \pm 0.12$ & - & 0.001 & 0.9785 \\
\hline & NADA & $2.977 \pm 0.46$ & $3.162 \pm 0.35$ & - & 0.104 & 0.7579 \\
\hline & DA & $0.791 \pm 0.14$ & $0.900 \pm 0.05$ & - & 0.542 & 0.4893 \\
\hline & $5 \mathrm{HT}$ & $1.588 \pm 0.26$ & $1.698 \pm 0.25$ & - & 0.093 & 0.7706 \\
\hline \multirow[t]{4}{*}{ 19th } & $\mathrm{OA}$ & $2.323 \pm 0.32(5)$ & $2.348 \pm 0.28(5)$ & $1.684 \pm 0.20$ & 1.887 & 0.1939 \\
\hline & NADA & $1.693 \pm 0.41(5)$ & $0.964 \pm 0.33(5)$ & $0.787 \pm 0.34(5)$ & 3.885 & 0.2167 \\
\hline & DA & $0.877 \pm 0.10$ & $0.771 \pm 0.11$ & $0.637 \pm 0.11$ & 1.319 & 0.3036 \\
\hline & $5 \mathrm{HT}$ & $1.001 \pm 0.22(5)$ & $0.648 \pm 0.16(5)$ & $0.521 \pm 0.09$ & 2.291 & 0.1437 \\
\hline
\end{tabular}

Data on the strains are presented as the mean \pm standard error, with the number in parenthesis indicating the sample size

$O A$ Octopamine, NADA $\mathrm{N}$-acetyldopamine, $D A$ dopamine, 5HT 5-hydroxytryptamine

${ }^{a} L W$ Strain selected by artificial selection for long-distance walking; $S W$ strain selected by artificial selection for short-distance walking

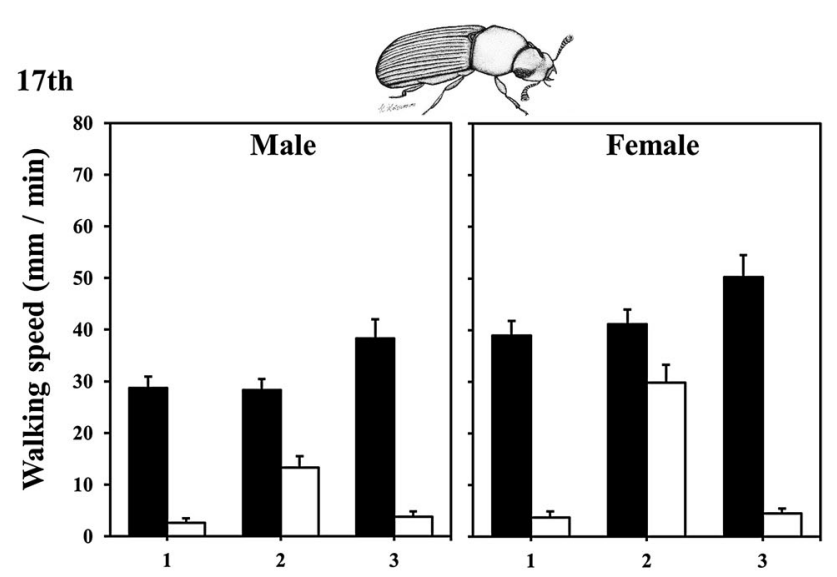

18th

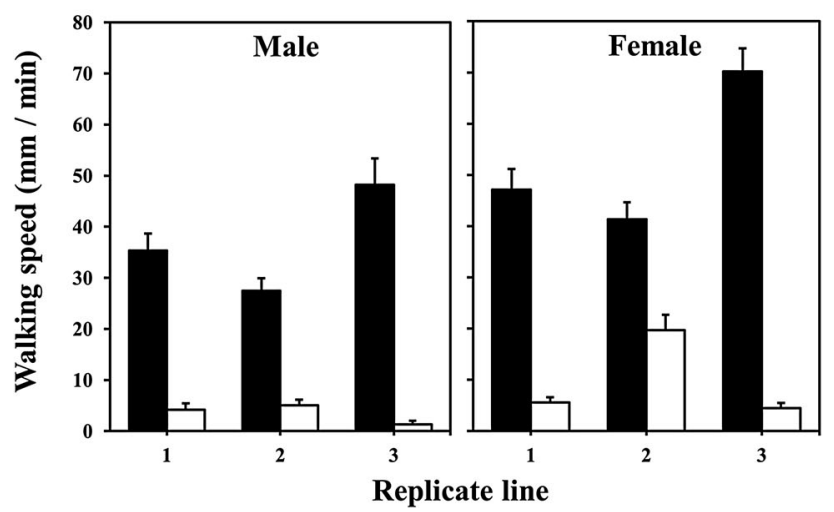

Fig. 5 Walking speed of T. castaneum LW (filled bars) and SW strain (open bars) at 17 th and 18 th generations. Error bars indicate SE

\section{Discussion}

Selection experiments are one approach that can be used to investigate the genetic base of traits (Hill and Caballero 1992), and an artificial selection experiment is one tool that is used to detect a genetic correlation (Ohno and Miyatake 2007). If a targeted selection which acts on trait A causes a correlated response in trait $\mathrm{B}$, and another selection which acts on trait B causes a correlated response in trait A, then a genetic link is considered to exist between traits A and B (Falconer and Mackay 1996; Lynch and Walsh 1998). Although some studies have demonstrated a genetic correlation between two behavioral traits by artificial selection in laboratory rodents (e.g. Careau et al. 2012; Waters et al. 2013), few such studies have been conducted in insects (but see Ohno and Miyatake 2007). Miyatake et al. (2008a) carried out artificial selection for longer and shorter duration of death-feigning behavior (LD and SD strain, respectively) in $T$. castaneum and observed that the walking dispersal distance was negatively correlated with the selection regime. In the study reported here, we carried out artificial selection for walking dispersal distance in $T$. castaneum and observed that the duration of death-feigning behavior was also negatively correlated with the selection regime. Based on the findings of these two studies, we suggest that dispersal ability and death-feigning behavior is genetically correlated in $T$. castaneum.

Individuals derived from the strains selected for higher dispersal ability had significantly higher locomotor activity than that of strains selected for lower dispersal ability. Although this result was expected, it is important that the findings of the present study suggest a genetic correlation between walking distance and locomotor activity. Previous studies have reported a trade-off between duration of death-feigning behavior and locomotor activity selected by artificial selection for duration of death-feigning in $T$. confusum and the adzuki bean beetle Callosobruchus chinensis (Nakayama and Miyatake 2010; Nakayama et al. 2010). The results of the present study suggest that the relationship between the three behavioral traits (dispersal, 
death-feigning, and locomotor activity) are genetically correlated with each other. To evaluate this hypothesis, additional studies need to be performed, including artificial selection for locomotor activity in $T$. castaneum and investigation of the dispersal ability and death-feigning behavior of the beetles from the strains selected as correlated responses.

Miyatake et al. (2008a) reported that an individually derived $T$. castaneum $\mathrm{LD}$ strain had a lower expression of brain dopamine than an individually derived $T$. castaneum strain. Nakayama et al. (2012) also reported similar results for Tribolium confusum. These results suggest that the death-feigning is genetically correlated with the expression of dopamine in the brain of Tribolium beetles. Because our results revealed a genetic correlation between dispersal and death-feigning, we expected that brain dopamine expression would correlate positively with selection for dispersal ability. However, our results showed that the expression of brain dopamine was not significantly different between selected strains and, in addition, that was no significant difference in the expression of the other biogenic amines evaluated between the selected strains. Although there is a possibility that the statistic power of our analysis was insufficient, these results suggest that dispersal ability is not correlated with the expression of biogenic amines. There are a number of possible explanations for these differences between the earlier studies and the present study.

One plausible explanation is the difference in the intensity of death-feigning. Intensity of death-feigning can be evaluated by the frequencies of death-feigning (Miyatake 2001). In our study, the frequency of death-feigning in the T. castaneum LW and SW strains was 70.00 and $95.33 \%$, respectively, and the duration of death-feigning of LW and SW strains was 38.51 and $191.65 \mathrm{~s}$, respectively, at the 17 th generation. In the study by Miyatake et al. (2008a), which involved artificial selection for duration of death-feigning using the same beetle, the frequency of death-feigning of LD and SD strains was about $100 \%$ and about $10 \%$, respectively, and the duration of death-feigning of LD and SD strains was about 1000 and about $0.1 \mathrm{~s}$, respectively, at the 17th generation (see Fig. 1 in Miyatake et al. 2008a). Specifically, the intensity of death-feigning (frequency and duration) observed in the present study is lower than that observed by Miyatake et al. (2008a). In some beetles, the intensity of death-feigning often varies in a natural population. This phenomenon suggests that death-feigning has depth and that this difference in "depth of death feigning" is included in the variation of death-feigning in a natural population (Miyatake 2001; Kuriwada et al. 2009). Therefore, it is possible that the selection pressure for death-feigning behavior of $T$. castaneum in Miyatake et al. (2008a) was larger than that in the present study; in other words, the depth of deathfeigning in the selected strains of the present study can be presumed to be shallow, possibly causing the observed lack of a significant difference in the expression of biogenic amines between selected strains.

However, the direct response in walking distance observed in the present study is clearly the same as that observed by Miyatake et al. (2008a), also suggesting that the selection regime in the present study was not sufficient to detect clear differences in biogenic amines between the selected strains. If the relationship between death-feigning behavior and biogenic amines is stronger than the that between dispersal and biogenic amines, the results might differ in the two studies.

Another possible explanation is that this selection favors traits other rather than biogenic amines. Other factors may influence the selection, such as characters in the peripheral nervous system, including neurotransmitters at the neuromuscular junction. Also, although the artificial selection for death-feigning behavior might directly target the neuronal circuitry between stimulations to activate the behavior and increase the persistence of death-feigning behavior, the artificial selection for dispersal ability might target the neuronal circuitry relating to persistence of locomotion. Additional studies are required to solve these problems.

The walking speeds of beetles of the LW strains were higher than those of the SW strains. This result lends to the supposition that dispersers adopt an escape by running tactics due to their higher moving ability when they encounter a predator. In many animals, escaping by running from predators is a common tactic and may be related to the level of locomotor activity (Edmunds 1974; Ducey and Brodie 1983; Cooper and Frederick 2010). For survival, the dispersers with higher moving ability and higher locomotor activity may adopt escaping behavior-rather than death-feigning behavior-as a tactic. Conversely, individuals with lower dispersal ability may adopt deathfeigning because they have lower moving ability and lower locomotor activity. Matsumura and Miyatake (2015) reported that $T$. castaneum SW strain beetles had an increased survival following encounters with A. venator, leading the authors to suggest that a higher intensity of death-feigning has an effect on the adaptive anti-predator strategy against $A$. venator. To clarify these issues, additional studies on the effect of death-feigning behavior or escaping by running from $A$. venator in the near future are needed.

The duration of death-feigning in females was shorter than that shown by males, and dispersal distances of females were longer than those of males. Females of the cowpea weevil Callosohrucbus maculatus also showed a longer duration of death-feigning behavior than males (Miyatake et al. 2008b). In the adzuki bean beetle 
Callosobruchus chinensis, although sex differences were not found in duration of death-feigning, the frequency of death-feigning in females was higher than that of males (Miyatake et al. 2008b). Further, the locomotor activity of females has been found to be lower than that of males in $C$. chinensis (Nakayama and Miyatake 2009). In T. castaneum, the duration of death-feigning in females appeared to be shorter than that of males (see Fig. 1 in Miyatake et al. 2008a). In contrast, no sex difference in the duration of death-feigning was observed in T. confusum (Nakayama et al. 2010) and Cylas formicarius (Miyatake 2001).

In conclusion, it would appear that differences in the intensity of death-feigning behavior and moving activity according to sex is species-dependent. C. formicarius females have a reduced of duration of death-feigning after copulation (Kuriwada et al. 2009), but we did not consider this effect in our study because we used only virgin females. It is necessary to examine the relationship between sex and death-feigning behavior by more detailed investigations.

Acknowledgments This work was supported by a grant from the Japan Society for the Promotion of Science KAKENHI 26291091 to T. M.

\section{References}

Aukema BH, Raffa KF (2004) Does aggregation benefit bark beetles by diluting predation? Links between a group-colonisation strategy and the absence of emergent multiple predator effects. Ecol Entomol 29:129-138

Bicker G, Menzel R (1989) Chemical codes for the control of behaviour in arthropods. Nature 337:33-39

Bonte D, Van Dyck H, Bullock JM, Coulon A, Delgado M, Gibbs M, Lehouck V, Matthysen E, Mustin K, Saastamoinen M, Schtickzelle N, Stevens VM, Vandewoestijne S, Baguette M, Barton K, Benton TG, Chaput-Bardy A, Clobert J, Dytham C, Hovestadt T, Meier CM, Palmer SCF, Turlure C, Travis JMJ (2012) Costs of dispersal. Biol Rev Camb Philos Soc 87:290-312

Careau V, Bininda-Emonds ORP, Ordonez G, Garland T Jr (2012) Are voluntary wheel running and open-field behavior correlated in mice? Different answers from comparative and artificial selection approaches. Behav Genet 42:830-844

Clobert J, Le Galliard JF, Cote J, Meylan S, Massot M (2009) Informed dispersal, heterogeneity in animal dispersal syndromes and the dynamics of spatially structured populations. Ecol Lett 12:197-209

Cooper WE Jr, Frederick WG (2010) Predator lethality, optimal escape behavior, and autotomy. Behav Ecol 21:91-96

Ducey PK, Brodie ED Jr (1983) Salamanders respond selectively to contacts with snakes: survival advantage of alternative antipredator strategies. Copeia 4:1036-1041

Edmunds M (1974) Defense in animals. Longman, London

Evans PD (1980) Biogenic amines in the insect nervous system. Adv Insect Physiol 15:317-473

Falconer DS, Mackay TFC (1996) Introduction to quantitative genetics, 4th edn. Longman, Harlow
Frazzetta T (1975) Complex adaptations in evolving populations. Sinauer, Sunderland

Hill WG, Caballero A (1992) Artificial selection experiments. Annu Rev Ecol 23:287-310

Korb J, Linsenmair KE (2002) Evaluation of predation risk in the collectively foraging termite Macrotermes bellicosus. Insectes Soc 49:264-269

Kuriwada T, Kumano N, Shiromoto K, Haraguchi D (2009) Copulation reduces the duration of death-feigning behavior in the sweetpotato weevil, Cylas formicarius. Anim Behav 78:1145-1151

Lehtiniemi M (2005) Swin or hide: predator cues cause species specific reactions in young fish larvae. J Fish Biol 66:1285-1299

Lima SL, Dill LM (1990) Behavioral decisions made under the risk of predation: a review and prospectus. Can J Zool 68:619-640

Lynch M, Walsh JB (1998) Genetics and analysis of quantitative traits. Sinauer Associates, Sunderland

Martin J, Lopez P (2000) Costs of refuge use affect escape decisions of Iberian rock lizards Lacerta monticola. Ethology 106:483492

Matsumura K, Miyatake T (2015) Differences in attack avoidance and mating success between strains artificially selected for dispersal distance in Tribolium castaneum. PLoS ONE 10(5):e0127042

Matsuyama S, Nagao T, Sasaki K (2015) Consumption of tyrosine in royal jelly increases brain levels of dopamine and tyramine and promotes transition from normal to reproductive workers in quenless honey bee colonies. Gen Comp Endocrinol 211:1-8

Miyatake T (2001) Diurnal periodicity of death-feigning in Cylas formicarius (Coleoptera: Brentidae). J Insect Behav 14:421-432

Miyatake T, Katayama K, Takeda Y, Nakashima A, Sugita A, Mizumoto M (2004) Is death-feigning adaptive? Heritable variation in fitness difference of death-feigning behaviour. Proc $\mathrm{R}$ Soc B 271:2293-2296

Miyatake T, Tabuchi K, Sasaki K, Okada K, Katayama K, Moriya S (2008a) Pleiotropic anti-predator strategies, fleeing and feigning death, correlated with dopamine levels in Tribolium castaneum. Anim Behav 75:113-121

Miyatake T, Okada K, Harano T (2008b) Negative relationship between ambient temperature and death-feigning intensity in adults Callosobruchus maculatus and Callosobruchus shinensis. Physiol Entomol 33:83-88

Nakayama S, Miyatake T (2009) A behavioral syndrome in the adzuki bean beetle: genetic correlation among death feigning, activity, and mating behavior. Ethology 116:108-112

Nakayama S, Miyatake T (2010) A behavioral syndrome in the adzuki bean beetle: genetic correlation among death feigning, activity, and mating behavior. Ethology 116:108-112

Nakayama S, Nishi Y, Miyatake T (2010) Genetic correlation between behavioural traits in relation to death-feigning behaviour. Popul Ecol 52:329-335

Nakayama S, Sasaki K, Matsumura K, Lewis Z, Miyatake T (2012) Dopaminergic system as the mechanism underlying personality in a beetle. J Insec Physiol 58:750-755

Ohno T, Miyatake T (2007) Drop or fly? Negative genetic correlation between death-feigning intensity and flying ability as alternative anti-predator strategies. Proc R Soc B 274:555-560

Roff DA, Fairbairn DJ (2007) The evolution and genetics of migration in insects. Bioscience 57:155-164

Ruxton GD, Sherratt TN, Speed MP (2004) Avoiding attack: the evolutionary ecology of crypsis, warning signals, and mimicry. Oxford University Press, Oxford

SAS Institute Inc (2007) JMP 7. SAS Institute Inc., Cary

Sasaki K, Yamasaki K, Nagao T (2007) Neuro-endocrine correlates of ovarian development and egg-laying behaviors in the primitively eusocial wasp (Polistes chinensis). J Insect Physiol 53:940-949 
Srygley RB (2004) The aerodynamic costs of warning signals in palatable mimetic butterflies and their distasteful models. Proc R Soc B Biol Sci 271:589-594

Stevenson PA, Hofmann HA, Schoch K, Schildberger K (2000) The fight and flight responses of crickets depleted of biogenic amines. J Neurobiol 43:107-120
Wasson K, Lyon BE (2005) Flight or fight: flexible antipredatory strategies in porcelain crabs. Behav Ecol 16:1037-1041

Waters RP, Pringle RB, Forster GL, Renner KJ, Malisch JL, Garland T Jr, Swallow JG (2013) Selection for increased voluntary wheel-running affects behavior and brain monoamines in mice. Brain Res 1508:9-22 